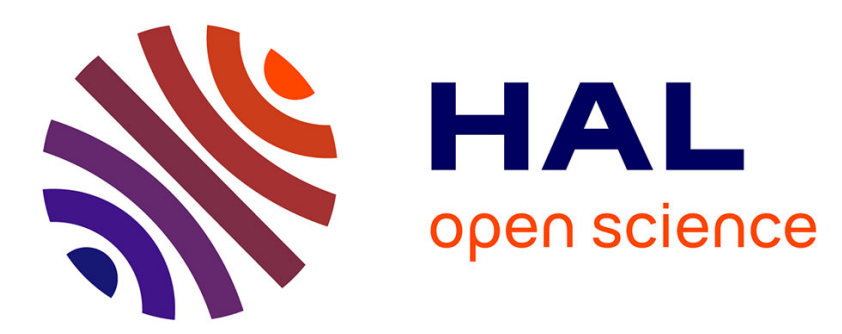

\title{
Dynamical selective ltering for the Lattice Boltzmann Method
}

\author{
Simon Marié, Xavier Gloerfelt
}

\section{To cite this version:}

Simon Marié, Xavier Gloerfelt. Dynamical selective ltering for the Lattice Boltzmann Method. 22nd AIAA Computational Fluid Dynamics Conference, Jun 2015, Dallas, United States. pp.1-8, 10.2514/6.2015-2454 . hal-01229632

\section{HAL Id: hal-01229632 \\ https://hal.science/hal-01229632}

Submitted on 17 Nov 2015

HAL is a multi-disciplinary open access archive for the deposit and dissemination of scientific research documents, whether they are published or not. The documents may come from teaching and research institutions in France or abroad, or from public or private research centers.
L'archive ouverte pluridisciplinaire HAL, est destinée au dépôt et à la diffusion de documents scientifiques de niveau recherche, publiés ou non, émanant des établissements d'enseignement et de recherche français ou étrangers, des laboratoires publics ou privés. 


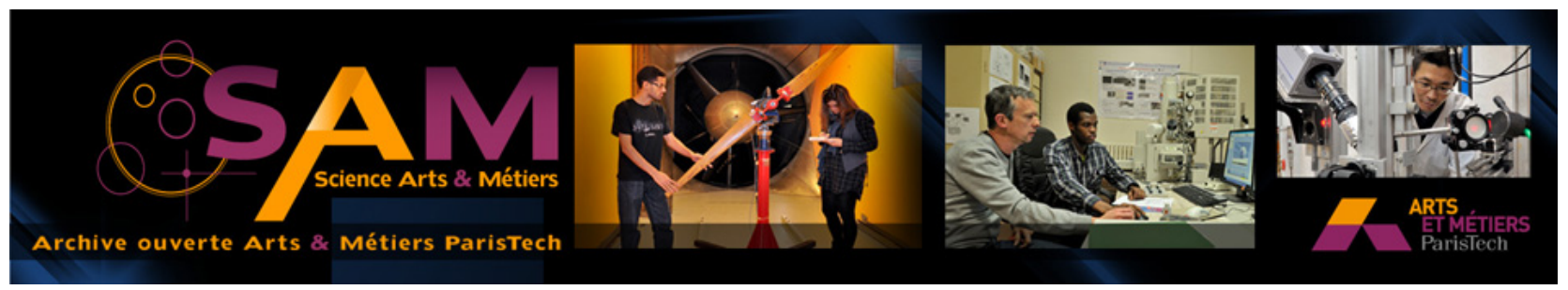

\section{Science Arts \& Métiers (SAM)}

is an open access repository that collects the work of Arts et Métiers ParisTech researchers and makes it freely available over the web where possible.

This is an author-deposited version published in: http://sam.ensam.eu

Handle ID: .http://hdl.handle.net/10985/10416

\section{To cite this version :}

Simon MARIÉ, Xavier GLOERFELT - Dynamical selective Itering for the Lattice Boltzmann Method - In: 22nd AIAA Computational Fluid Dynamics Conference, Etats-Unis, 2015-06-22 22nd AIAA Computational Fluid Dynamics Conference - 2015 


\title{
Dynamical selective filtering for the Lattice Boltzmann Method
}

\author{
S.Marié* and X. Gloerfelt ${ }^{\dagger}$ \\ DynFluid laboratory, 151 boulevard de l'Hopital, 75013 Paris, France.
}

\begin{abstract}
In this study, a new selective filtering technique is proposed for the Lattice Boltzmann Method. This technique is based on dynamical implementation of the selective filter coefficient $\sigma$. The proposed model makes the latter coefficient dependent on the shear stress in order to restrict the use of the spatial filtering technique in the sheared stress region where numerical instabilities may occur. Different parameters are tested on a $3 D$ decaying Taylor Green Vortex and compared to the classical static filtering technique and to the use of a standard subgrid-scale model.
\end{abstract}

\section{Introduction}

The Lattice Boltzmann Method ${ }^{12}$ (LBM) is used to simulate the behavior of weakly compressible flows. Some studies ${ }^{3}$ have shown the capabilities of LBM to perform aeroacoustic simulations due to its low dissipation error. However, as a counterpart, LBM suffers from numerical instabilities when Reynolds number becomes high. Moreover, numerical instability waves are often generated by unadapted initial conditions, geometric singularities or in region where large numerical approximations are done. In industrial applications several of these numerical defaults are present, thus computations often become catastrophically unstable.

Various studies have proposed stabilization techniques. ${ }^{4-7}$ Lots of stabilizing strategies have a global effect on the viscosity thus modifying the effective Reynolds number. Another effect of stabilizing methods is to increase the global dissipation then impacting pressure fluctuation from which it could be important to keep an accurate estimation (e.g. for aeroacoustic simulations). Ricot et al. ${ }^{7}$ proposed to use selective spatial filters ${ }^{8}$ to stabilize the method by increasing the dissipation for high wavenumbers where the LBM instabilities occur and then keeping a low dissipation at small wavenumbers. This approach can be applied to aeroacoustic simulations by maintaining an acceptable level of dissipation error at low wavenumbers. However, this method basically applies to the whole domain and becomes useless outside of sheared region where numerical instabilities have less chances to develop. Furthermore, the use of high-order filters have the effect of increasing the stencil of LBM which is low by nature and lead to a loss of locality of the method which is known to be one of the main advantages of the method. Finally, from a dynamical point of view, the selective spatial filters have never been tested on transition situation where the dynamical evolution prediction is of major importance for accuracy of the results. Therefore, the need for improved stabilization procedure is justified and should be carried out, in particular in the framework of the Lattice Boltzmann Method.

The idea of the present study is to propose an improved filtering strategy restricted to highly sheared region keeping low-sheared ones free of artificial dissipation. The proposed strategy is developed in the framework of the Lattice Boltzmann Method and applied to a dynamical test-case highly sensitive to the numerical dissipation. After a brief presentation of the Lattice Boltzmann model, the new filtering strategy is described and validated on a 3D decaying Taylor Green Vortex and then compared to the usual filtering techniques.

\footnotetext{
* Assistant Professor, Conservatoire National des Arts et Métiers.

${ }^{\dagger}$ Professor, Ecole Nationale Supérieure des Arts et Métiers.
} 


\section{Model}

\section{II.A. Lattice Boltzmann Method}

The Lattice Boltzmann method ${ }^{1}$ is described by the following algorithm:

$$
g_{\alpha}\left(\mathbf{x}+\mathbf{c}_{\alpha}, t+1\right)=g_{\alpha}(\mathbf{x}, t)-\frac{1}{\tau_{g}}\left[g_{\alpha}(\mathbf{x}, t)-g_{\alpha}^{e q}(\mathbf{x}, t)\right]
$$

where $g_{\alpha}$ are distribution functions computed on a regular velocity lattice $c_{\alpha}$, colliding and relaxing to a local

equilibrium $g_{\alpha}^{e q}$ with a relaxing parameter $\tau_{g}=\frac{\nu}{\tilde{c}_{0}^{2}}+\frac{1}{2}$ where $\nu$ and $\tilde{c}_{0}$ being the adimensional viscosity and speed of sound respectively. In this study, we use the D3Q19 model described by the following parameters:

$$
\begin{gathered}
g_{\alpha}^{e q}(\mathbf{x}, t)=\rho \omega_{\alpha}\left(1+\frac{\mathbf{u} \cdot \mathbf{c}_{\alpha}}{\tilde{c}_{0}^{2}}+\frac{\left(\mathbf{u . c}_{\alpha}\right)^{2}}{2 \tilde{c}_{0}^{4}}-\frac{|\mathbf{u}|^{2}}{2 \tilde{c}_{0}^{2}}\right) \\
\omega_{\alpha}=\left\{\begin{array}{ccc}
\frac{1}{3} \quad, & \alpha=0 \\
\frac{1}{18} \quad, \quad \alpha=1 \rightarrow 6 & \tilde{c}_{0}^{2}=\frac{1}{3} \\
\frac{1}{36} & , \quad \alpha=7 \rightarrow 18
\end{array}\right.
\end{gathered}
$$

It should be noticed that the construction of Eq.(1) enforces the space and time discretization to be linked by the relation:

$$
\Delta t=\frac{\tilde{c}_{0} \Delta x}{c_{0}}
$$

This enforces the CFL number $\left(c_{0} \Delta t / \Delta x\right)$ to be the same for each simulations $\left(\mathrm{CFL}=\tilde{c}_{0}\right)$. The macroscopic quantities $\rho$ and $\mathbf{u}$ can be computed from the distribution functions with the discrete momenta:

$$
\begin{gathered}
\rho=\sum_{\alpha} f_{\alpha} \\
\rho \mathbf{u}=\sum_{\alpha} \mathbf{c}_{\alpha} f_{\alpha}
\end{gathered}
$$

The pressure is recovered by the relation:

$$
p=\tilde{c}_{0}^{2} \rho
$$

Based on these parameters, it can be shown ${ }^{2}$ that LBM simulates the 3D compressible Navier-Stokes equations in the limit of low Mach numbers with a $2 n d$ order accuracy in space and time.

\section{II.B. Dynamical selective spatial filters}

As proposed by Ricot et al., ${ }^{7}$ the stability of LBM can be enhanced by space-filtering the momenta of eq.(5) and (6). Spatial filtering of a quantity $Q$ is defined by subtracting a weighted combination of the symmetric neighboring points:

$$
\langle Q(\mathbf{x})\rangle=Q(\mathbf{x})-\sigma \sum_{j=1}^{D} \sum_{n=-N}^{N} d_{n} Q\left(\mathbf{x}+n \Delta x_{j}\right)
$$

where $\sigma$ is a coefficient between 0 and 1 often taken to $0.1, d_{n}$ are coefficients depending on the filter order and $D$ is the number of spatial dimension. In this study, classical 3-point, 5-point stencil and optimized 9-point stencil filters are used. ${ }^{8}$ The coefficients of the filters are summarized in table 1.

The idea of this study is to make the coefficient $\sigma$ dependent on the shear stress. For instance, let's consider $\sigma_{d}(\mathbf{x})$ to be of the form:

$$
\sigma_{d}(\mathbf{x})=\sigma_{0}\left(1-e^{-\left(|S(\mathbf{x})| / S_{0}\right)^{2}}\right)^{2}
$$

$$
2 \text { of } 8
$$




$\begin{array}{cccccc} & d_{0} & d_{1} & d_{2} & d_{3} & d_{4} \\ \text { SF-3 } & 1 / 2 & -1 / 4 & & & \\ \text { SF-5 } & 6 / 16 & -4 / 16 & 1 / 16 & & \\ \text { Bogey-9 (B9) } & 0.243527493120 & -0.204788880640 & 0.120007591680 & -0.045211119360 & 0.008228661760\end{array}$

Table 1. Coefficients of the selective filters: ${ }^{8} d_{n}=d_{-n}$.

where $|S|=\sqrt{2 S_{i j} S_{i j}}, \sigma_{0}$ is the static filter amplitude and $S_{0}$ is the shear stress amount from which the dynamical filter start to be active. Thus, when the shear stress is low, the filter has no effect $\left(\sigma_{d} \sim 0\right)$ and when shear stress rises to higher values, the filter acts normally $\left(\sigma_{d} \sim \sigma_{0}\right)$. Then $S_{0}$ should be seen as a sensitivity parameter and its influence will be further discussed in section III.B.2. The quantity $|S|$ involves spatial derivatives that must be computed during the simulations. From a Lattice Boltzmann point of view, this quantity could be computed from the second order momentum:

$$
\tau_{i j}=2 \rho \nu S_{i j}=-\sum_{\alpha} c_{\alpha, i} c_{\alpha, j}\left(g_{\alpha}-g_{\alpha}^{e q}\right)
$$

which gives:

$$
|S|=\frac{\mathbf{Q}_{\mathbf{f}}}{2 \rho \nu}
$$

with $\mathbf{Q}_{\mathbf{f}}=\sqrt{2 P_{i j} P_{i j}}$ and $P_{i j}=\sum_{\alpha} c_{\alpha, i} c_{\alpha, j}\left(g_{\alpha}-g_{\alpha}^{e q}\right)$. Relation (11) is often use for the implementation of subgrid scale models in the Lattice Boltzmann Method. ${ }^{9}$

In the following, the present model is tested on a 3D decaying Taylor-Green vortex and compared to the classical static filters and to the Smagorinsky subgrid-scale model.

\section{Taylor Green Vortex}

\section{III.A. Test case implementation}

In order to study the effect of the present filtering technique, the decaying Taylor-Green vortex is used. It is a fundamental case used as prototype for vortex stretching and production of small-scale eddies and therefore allows the study of the dynamics of transition to turbulence. This test-case has been widely used to study the dissipation errors of numerical schemes. ${ }^{10}$ In particular, Aubard et al. ${ }^{11}$ have recently used this test-case to confront the selective filtering techniques to the use of subgrid-scale models.

The initialization of the Taylor-Green vortex is done by setting velocity and pressure variables as follows:

$$
\left\{\begin{aligned}
p & =p_{\infty}+\frac{\rho_{\infty} U_{\infty}^{2}}{16}[\cos (2 z)+2][\cos (2 x)+\cos (2 y)] \\
u & =U_{\infty} \sin (x) \cos (y) \cos (z) \\
v & =-U_{\infty} \cos (x) \sin (y) \cos (z) \\
w & =0
\end{aligned}\right.
$$

In order to reduce numerical oscillations at the beginning of the simulation, the distribution functions $g_{\alpha}$ are initialized to their equilibrium state with an additional non-equilibrium part based on the ChapmanEnskog micro-scale expansion. More discussions about LBM initialization are detailed in Skordos. ${ }^{12}$

For this study, the Mach number is taken to $M_{\infty}=0.085$ and $\rho_{\infty}=1$ fixing the other parameters to $U_{\infty}=0.049$ and $p_{\infty}=1 / 3$ in lattice unit. The relaxation parameter is set with the Reynolds number $R e$ to $\tau_{g}=\frac{U_{\infty}}{\Delta x \tilde{c}_{0}^{2} R e}+\frac{1}{2}$. For the validation of this test case, the spectral data from Brachet et al. ${ }^{13}$ are used and compared to our reference simulation on a $256^{3}$ grid. 


\section{III.B. Results and discussion}

All the simulations of this study are performed on a $2 \pi$-periodic cubic domain with a Reynolds number of $R e=1600$. A reference simulation is performed on a $256^{3}$ grid without any filtering technique. Figure 1 displays the evolution of the sheared quantities in the domain. The classical behaviour of the Taylor-Green vortex is observed, the initial field gives rise to large vortices which are then stretched and lead to the production of small-scale eddies and decaying turbulence.
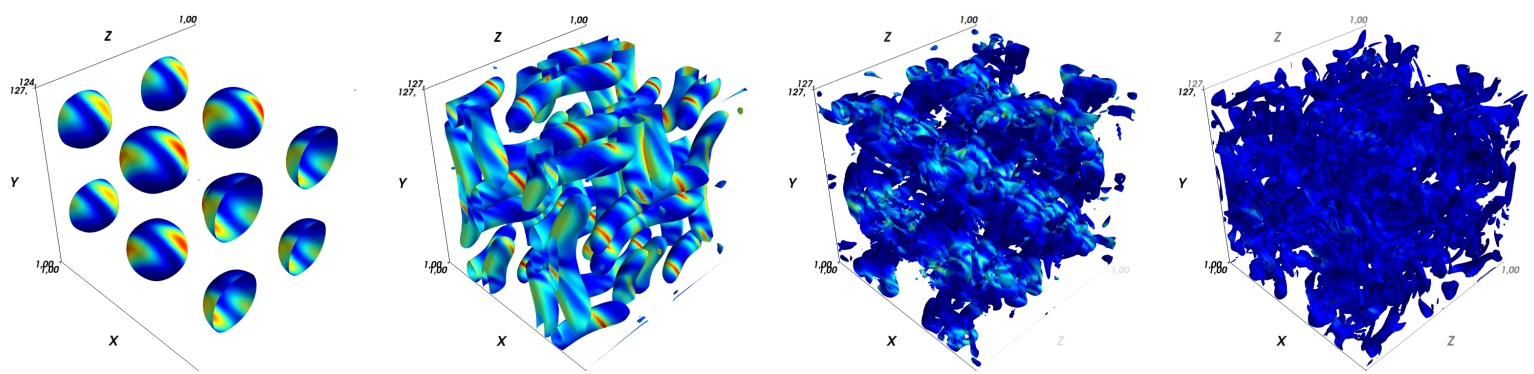

Figure 1. Isosurface of the Q-criterion colored by kinetic energy at time $t=0, t=4, t=10, t=16$ for $R e=1600$ on a $128^{3}$ grid.

In the following, a series of tests are performed to characterize the present filtering strategy. First, the influence of the grid resolution on the non-filtered scheme is presented, then the influence of parameters $\sigma_{0}$ and $S_{0}$ in equation (9) are scrutinized and finally, a comparison with the static filtering strategy and the subgrid-scale model is performed.

\section{III.B.1. Influence of the resolution}

The lattice Boltzmann method is a $2 n d$-order accuracy scheme in space and time. In the literature, few studies have been published on the validation of the 3D Taylor-Green vortex with LBM simulations. Then it could be interesting to see the capabilities of the classical scheme to simulates the dynamics of a $3 \mathrm{D}$ decaying vortex. For that purpose, the evolution of the kinetic energy dissipation rate $\epsilon=-\partial_{t} k$ is scrutinized for various resolution from $64^{3}$ to $256^{3}$ and compared with the spectral simulation of Brachet et al. ${ }^{13}$

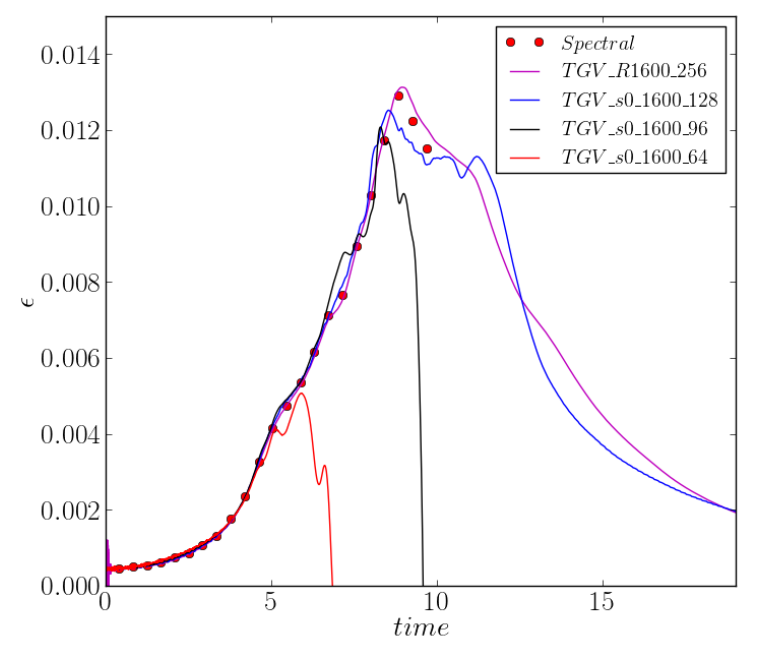

Figure 2. Non dimensional time evolution of the dissipation rate $\epsilon$.

The TGV dynamical evolution is characterized by three main steps visible in the time trace of $\epsilon$. First, the initial laminar state is transitioning to turbulence until the stretched vortex tubes break down into small-scales around $t=5$. Then the dissipation rate is rising to a sharp peak near $t=9$ corresponding to the full turbulent state which is then decaying similarly to an isotropic and homogeneous turbulence. 
The results of the classical LBM simulations without any filtering technique are displayed in figure 2 . The reference simulation on a $256^{3}$ grid is observed to be in very good agreement with the spectral results. The $128^{3}$ grid gives satisfactory results for the transition region but the peak in the dissipation rate is not fully reached and the decaying phase starts too early. Then the $64^{3}$ and $96^{3}$ simulations give rise to basic instability at different time. The $96^{3}$ grid simulation exhibits a relatively good transition to turbulence but collapses just before the peak of dissipation around $t=8$. Finally, the $64^{3}$ grid simulation collapses around $t=5$ when the stretched vortex tubes break down into small-scales.

Thus, the LBM scheme shows a good ability to simulate the Taylor-Green vortex dynamics when using fine grids but is limited by its inherent instability for coarser grids. Then the use of stabilizing filtering strategy becomes a crucial point to investigate turbulent simulation with the Lattice Boltzmann Method.

\section{III.B.2. Tuning parameters}

As described in section II.B, the dynamical filtering strategy is sensitive to two main parameters which are $\sigma_{0}$ and $S_{0}$. The first one determines the filtering amplitude when the shear stress is high and could be seen as the efficiency of the filter. The latter one plays the role of shear stress selectivity and is essential for controlling the dissipation amount of the filtering technique.

The sensitivity to these parameters is then investigated on a $96^{3}$ grid for various values of $\sigma_{0}, S_{0}$ and the different selective filters presented in table 1 . The nomenclature used to distinguish the simulation is based on a $s \mathbf{s} f \mathbf{f} d \mathbf{d}$ name where $\mathbf{s}=100 \sigma_{0}, \mathbf{f}$ is the number of points of the selective filter and $\mathbf{d}=100 S_{0}$. For example, the $s 5 f 3 d 100$ simulation refers to the use of the 3 -points filter with $\sigma_{0}=0.05$ and $S_{0}=1$ in lattice unit.
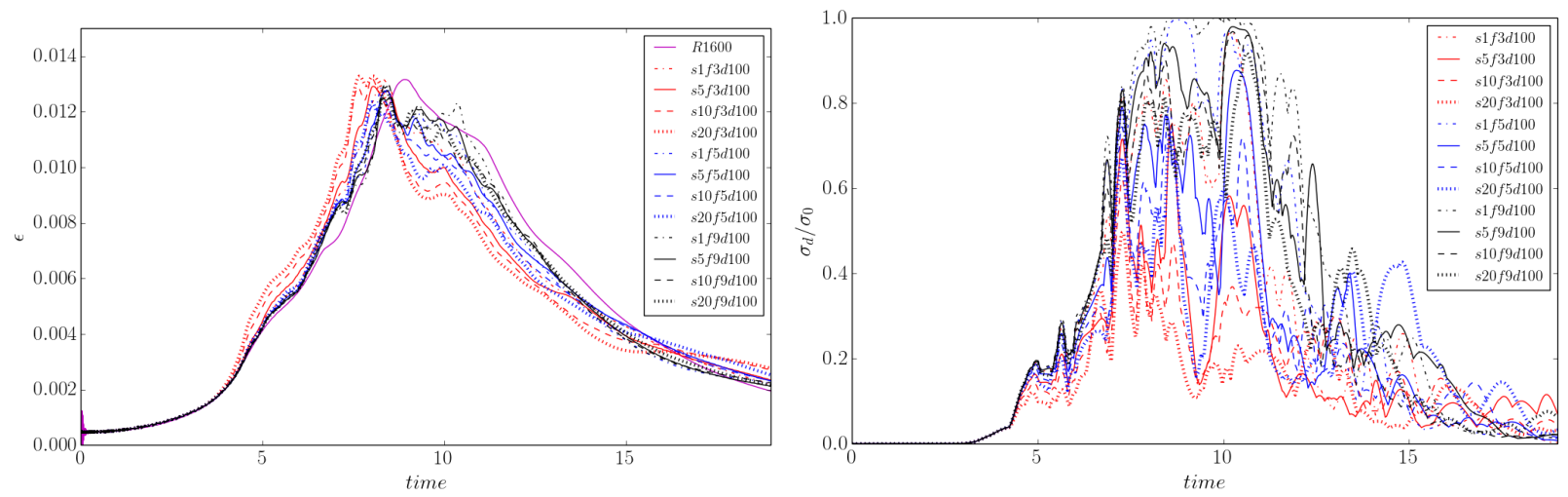

Figure 3. Influence of the $\sigma_{0}$ parameter. Non dimensional time evolution of the dissipation rate (left) and maximum value of normalized dynamic coefficient $\max \left[\sigma_{d}(\mathbf{x})\right] / \sigma_{0}$ (right).

A first sensitivity analysis is performed on the $\sigma_{0}$ parameter with a fixed value of $S_{0}=1$. Results are reported in figure 3-left and exhibit relatively similar behaviour. A high value of $\sigma_{0}$ (thick dotted lines) induces more damped results, in particular after the vortex breakdown near $t=5$ when the filter order is low (red curves). On the other hand, better results are obtained for low $\sigma_{0}$ values (thin lines). This improvement is particularly important for the 3-point filter which can handle the vortex breakdown around $t=5$ when $\sigma_{0}$ is set to 0.01. Lower values of $\sigma_{0}$ have been tested but lead to unstable simulations. This suggest that the dynamical filtering strategy should be applied for values of $\sigma_{0}$ as low as possible in the limit of stability. By looking at figure 3-right where the time evolution of $\sigma_{d}$ is represented, one can see that the value of $\sigma_{0}$ has a direct impact on the time evolution of $\sigma_{d}$. Indeed, when $\sigma_{0}$ changes, the filtering amount is modified and so is the shear stress which modifies the local $\sigma_{d}$ value. Moreover, for higher values of $\sigma_{0}$ the maximum value of $\sigma_{d}$ is never reached suggesting that the filtering amount induces lower shear stress and then reducing $\sigma_{d}$. As a counterpart, when $\sigma_{0}$ is low, the filtering amount allows larger shear stress values then increasing $\sigma_{d}$ to its maximum value fixed by $\sigma_{0}$. However, these considerations depends on the shear stress selectivity which is driven by the value of $S_{0}$.

The second sensitivity analysis is performed on the $S_{0}$ parameter with a fixed value of $\sigma_{0}=0.05$. Results are reported in figure 4-left. It shows that the selected $S_{0}$ values have an important impact on the results. A lower sensitivity (dashed lines) induces an earlier filtering which damp the initial laminar state and lead to 

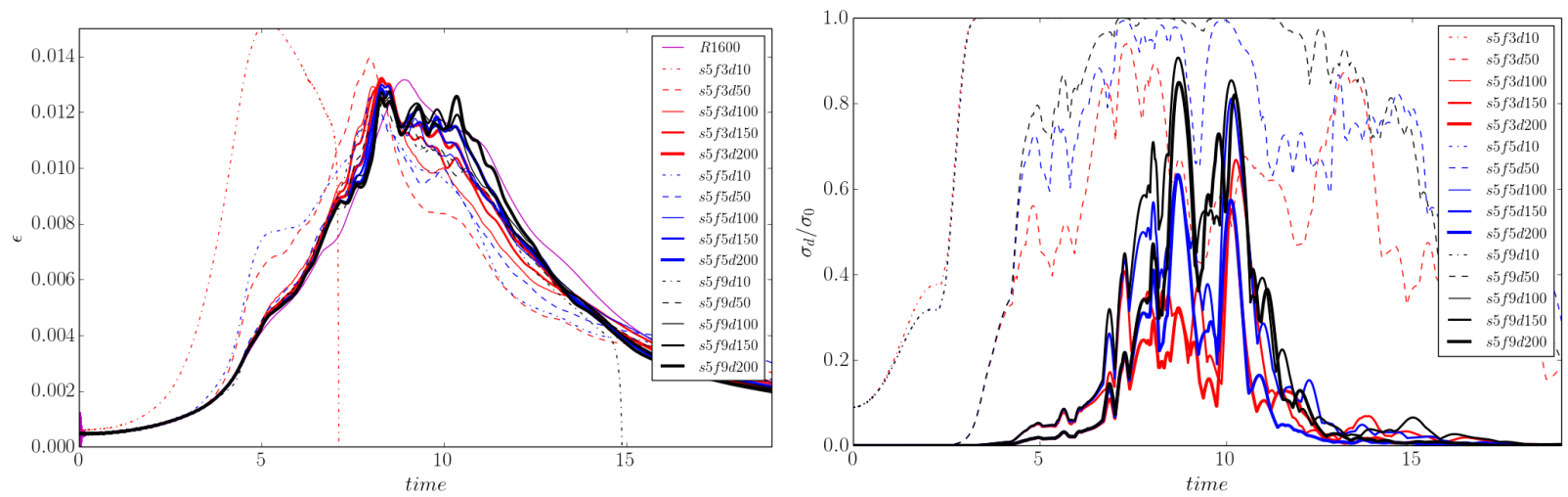

Figure 4. Influence of the $S_{0}$ parameter. Non dimensional time evolution of the dissipation rate (left) and maximum value of normalized dynamic coefficient $\max \left[\sigma_{d}(\mathbf{x})\right] / \sigma_{0}$ (right).

a wrong dissipation rate evolution. Indeed, results of figure 4-right indicates that $\sigma_{d}$ starts to increase from a not zero value for low sensitivity filters. In contrast, when the sensitivity is high (solid lines), the filter does not act in low sheared region and is activated only when large vortices have broken up and small-scales structures are rising to turbulence. Moreover it could be seen from figure 4 that the impact of the filter order is reduced when increasing the sensitivity. Then, 3-points filter results are close to these of the 9-point filter when sensitivity is high (thick solid lines). This is an important consequence in terms of computational cost suggesting that the filter order could be reduced when increasing sensitivity.

These tuning tests have emphasized the important role played by the couple $\left(\sigma_{0}, S_{0}\right)$ on the simulation of a 3D decaying Taylor-Green vortex. In the following simulations, this couple will be set to $(0.05,1$.$) .$

\section{III.B.3. Comparison with static filters}

The static filtering technique could be seen as a particular case of the dynamical one when the sensitivity is set to zero $\left(S_{0}=0\right)$. Then, static filters act everywhere with the same amount, with potential damping of important structures in the dynamical evolution. With the above discussion, the static filtering technique is then expected to give dissipated results. This is confirmed by looking at figure 5 which displays the comparison of static and dynamical filtering on a $96^{3}$ grid (left) and a $128^{3}$ grid (right).
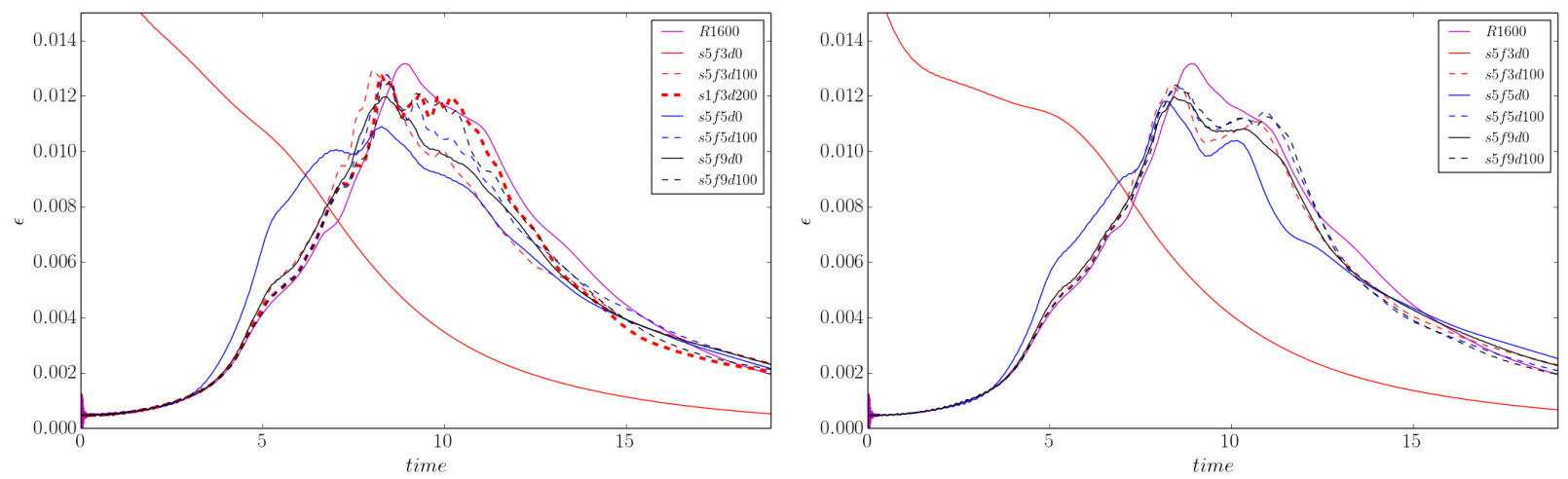

Figure 5. Comparison between static filters (solid lines) and dynamical filters (dashed lines) on a $96^{3}$ domain (left) and a $128^{3}$ domain (right).

An important result is observed for 3-point stencil filter which gives completely wrong behaviour with a static strategy and exhibits results close to higher order filters for the dynamical strategy. This is particularly observed in the transition region where 3-point dynamical filter gives better results than the static 5-point filter and similar results with the static 9-point filter. Moreover, the dynamical filtering appears to better predict the dissipation peak around $t=9$ than the static filters. The above discussions thus indicates that the results could be improved by increasing the filtering sensitivity and decreasing the $\sigma_{0}$. Then the $96^{3}$ grid 
simulation with $\sigma_{0}=0.01$ and $S_{0}=2$ is added for indication on figure 5-left and gives satisfactory results. This behaviour suggests that the wavenumber selectivity of the spatial filters plays a minor role compared to the shear stress selectivity. Indeed, the results indicates that applying a high-order wavenumber selectivity on non-sheared quantities (i.e. with static filters) could lead to over-dissipation in the transition region. As a counterpart, by introducing a shear-stress dependency on the filters, the wavenumber selectivity is forced to act on turbulent quantities with an important shear amount which corresponds to large-scale structures on which the wavenumber selectivity has no impact and could be reduced by decreasing the filter order. This result is of major importance in the framework of lattice Boltzmann method showing that relevant results could be obtained with a 3-point stencil selective filter which correspond to the LBM stencil, then conserving the locality of the scheme particularly important when dealing with heavy parallel implementations.

\section{III.B.4. Comparison with SGS models}

The Lattice Boltzmann implementation of subgrid-scale model is very close to the one of the present dynamical filtering. Relation (11) is used to estimate the eddy viscosity and thus the relaxation time $\tau_{g}$. The comparison between the implementation of the classical Smagorinsky model and the present methodology is presented on figure 6 where turb 1 refers to the Smagorinsky model with a constant $C_{s}=0.1$ and $\operatorname{turbSm}$ with a constant 0.18 . The results indicates that the SGS implementation appears to be too dissipative and describes poorly the laminar-turbulent transition region. Similar observations are detailed in Aubard et al. ${ }^{11}$ where various SGS strategy has been compared and found to be not adapted to this type of transition test case.
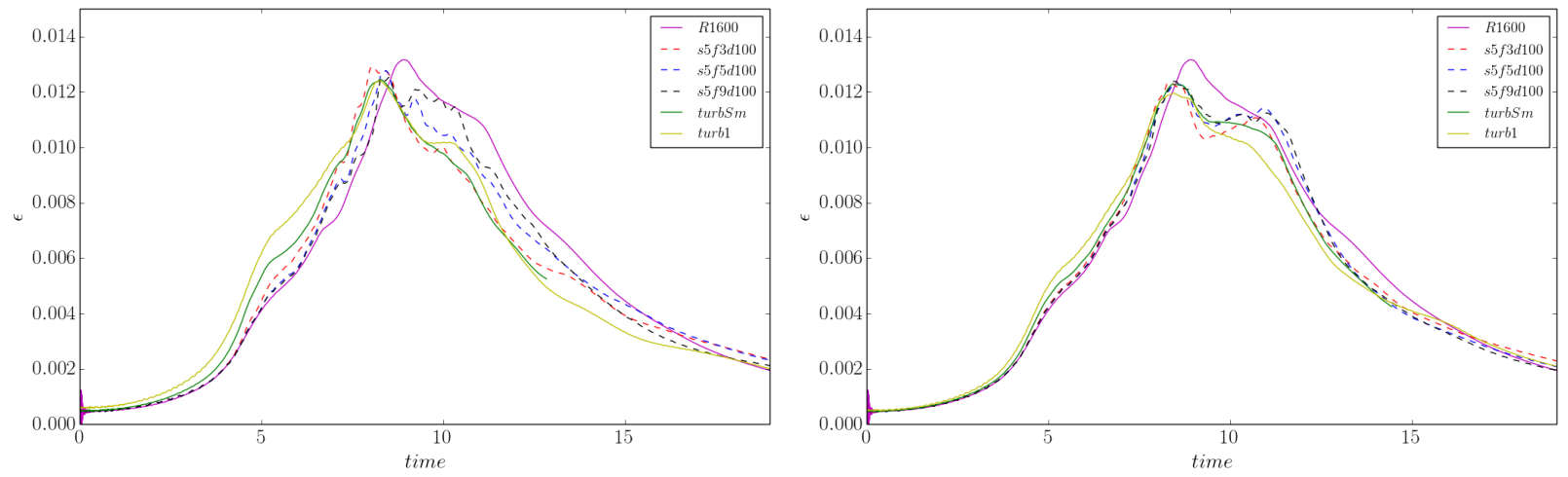

Figure 6. Comparison between dynamical filtering strategy and subgrid-scale model on a $96^{3}$ domain (left) and a $128^{3}$ domain (right).

The present dynamical strategy is found to better predict the transition region by filtering only the sheared region. The main difference between the present implementation and the SGS methodology relies in the amount of filtering when shear stress is lower than the imposed sensitivity $\left(S<S_{0}\right)$. Indeed for those values the filter have no impact and let the turbulent structures free of numerical dissipation. As a counterpart, the SGS implementation is directly proportional to the amount of shear stress and the eddy viscosity have a non zero value for shear stress amount close to $S_{0}$ thus imposing an over-dissipation for the concerned structures which are of primary importance for the dynamical evolution. Moreover, the SGS approach is not a bounded procedure and the eddy viscosity can reach arbitrary high value when highly sheared region are encountered whereas the presented strategy is limited by the $\sigma_{0}$ value restricting the numerical dissipation to a limited amount. Finally, from a general point of view, the SGS strategy could be seen as the present dynamical filtering technique with a low shear stress sensitivity and should be linked to the results of section III.B.2.

\section{Conclusion}

A dynamical filtering technique for the lattice Boltzmann method has been presented and tested on a 3D decaying Taylor-Green vortex. It has been shown that the use of selective spatial filters with a coefficient based on the amount of shear stress led to improved dynamical predictions of the 3D decaying Taylor-Green 
vortex. In particular, it has been emphasized that the shear stress selectivity was restricting the action of the filters to localized zones then reducing the global amount of numerical dissipation. The choice of shear stress selectivity has been motivated by the lattice Boltzmann framework for which the shear stress is a relevant quantity that can be accessed with a minimum amount of additional computational time. The results are particularly improved when the filter order is low, especially for the comparison with static selective filters suggesting that the wavenumber selectivity is dominated by the one of the shear stress. The comparison of the presented methodology to the classical subgrid-scale model methodology on a laminar-turbulent transition test case such as the Taylor-Green vortex have also led to promising results highlighting the importance of shear-stress selectivity in the prediction of the turbulence dynamical evolution. From a computational cost point of view, the presented strategy have shown interesting capabilities when using low-order filters thus reducing the effective stencil to the one of the LBM.

Then the dynamical filtering strategy should be seen as an enhanced stabilization procedure for the lattice Boltzmann method where the amount of numerical dissipation could be controlled in space. The presented methodology have been tested on a specific test-case and should be improved by making the sensitivity $S_{0}$ independent of the test case. Further studies must be carried out to overcome these limitations through a global stability analysis. Moreover, additional investigations must be carried out to characterize the dynamical filtering behaviour in the case of wall-bounded flows.

Finally it should be noticed that the presented methodology could be applied to a wide variety of numerical problems where only local dissipation is required. Moreover, the presented procedure should be extended to different physical problems by modifying the sensitive quantity and not restrict it to the shear stress. Furthermore, the presented dynamical filtering is not restricted to the Lattice Boltzmann method and could be applied to the classical finite differences schemes.

\section{References}

${ }^{1}$ Qian, Y.-H., d'Humières, D., and Lallemand, P., "Lattice BGK models for Navier-Stokes equation." Europhys.Lett., Vol. 17, 1992, pp. 479-484.

${ }^{2}$ He, X. and Luo, L.S., "A priori derivation of the lattice Boltzmann equation," Phys. Rev. E, Vol. 55, 1997, pp. R6333.

${ }^{3}$ Marié, S., Ricot, D., and Sagaut, P., "Comparison between Lattice Boltzmann Method and Navier-Stokes high order schemes for Computational Aeroacoustics." Journal of Computational Physics., Vol. 228, 2009, pp. 1056-1070.

${ }^{4}$ Sterling, J.D. and Chen, S., "Stability analysis of lattice Boltzmann methods," J. Comp. Phys., Vol. 123, 1996, pp. 196206.

${ }^{5}$ d'Humière, D., Ginzburg, I., Krafczyk, Y., Lallemand, P., and Luo, L., "Multiple relaxation time lattice Boltzmann models in three dimensions," Phil. Trans. R. Soc. Lon. A, Vol. 360, 2002, pp. 437-451.

${ }^{6}$ Dellar, P.J., "Bulk and shear viscosities in lattice Boltzmann equations," Phys. Rev.E, Vol. 64, 2003.

${ }^{7}$ Ricot, D., Marié, S., Sagaut, P., and Bailly, C., "Lattice Boltzmann Method with selective viscosity filters," Journal of Computational Physics., Vol. 228, 2009, pp. 4478-4490.

${ }^{8}$ Bogey, C. and Bailly, C., "A family of low dissipative explicit schemes for flow and noise computations," J. Comput. Phys., Vol. 194, 2004, pp. 194-214.

${ }^{9}$ Dong, Y., Sagaut, P., and Marié, S., "Inertial consistent subgrid model for Large Eddy Simulation based on Lattice Boltzmann Method." Physics of Fluids, Vol. 20, 2008.

${ }^{10}$ Fauconnier, D., Bogey, C., and Dick, E., "On the performance of relaxation filtering for large-eddy simulation," Journal of Turbulence, Vol. 14, 2013, pp. 22-49.

${ }^{11}$ Aubard, G., Stefanin Volpiani, P., Gloerfelt, X., and Robinet, J.-C., "Comparison of subgrid-scale viscosity models and selective filtering strategy for large-eddy simulations," Flow Turbulence Combustion, Vol. 91, No. 3, 2013, pp. $497-518$.

${ }^{12}$ Skordos, P., "Initial and Boundary Conditions for the lattice Boltzmann Method." Physical Review E, Vol. 48, No. 6, 1993, pp. 4823-4842.

${ }^{13}$ Brachet, M., Meiron, D., Orszag, S., Nickel, B., Morf, R., and Frisch, U., "Small-scale structure of the Taylor-Green vortex." J. Fluid Mech., Vol. 130, 1983, pp. 411-452. 